\title{
THE UV-OPTICAL-NEAR-IR EMISSION OF BL LACERTAE OBJECTS.
}

\author{
R. FALOMO \\ Osservatorio Astronomico di Padova, v. Osservatorio 5, 35122, Padova, Italy \\ E. PIAN \\ Scuola Internazionale Superiore di Studi Avanzati, via Beirut 2-4, 34014 Trieste, Italy \\ R. SCARPA \\ Dipartimento di Astronomia dell'Università di Padova, v. Osservatorio 5, 35122 Padova, Italy \\ and \\ A. TREVES \\ Scuola Internazionale Superiore di Studi Avanzati, via Beirut 2-4, 34014 Trieste, Italy

\begin{abstract}
Results from UV, optical and near-IR simultaneous observations for 11 BL Lac objects are reported. We find that for all but one source the spectral flux distribution can be described by a single power law $\left(f_{\nu} \propto \nu^{-\alpha}\right)$ plus, where relevant, the contribution of the host galaxy. The comparison of the optical-near-IR and UV spectral indices for two samples of BL Lacs suggests the same picture for a larger sample of objects.
\end{abstract}

\section{Introduction}

The overall spectral flux distribution (SFD) of BL Lac objects, is usually interpreted as due to the synchrotron or synchrotron self-Compton processes. Depending on the considered energy range, complex forms, like broken power laws or a curve that steepens with increasing frequency are used to describe the SFD rather than a single power law (e.g. Landau et al. 1986, Impey and Neugebauer 1988). Spectral "breaks" are reported to occur between near-IR and optical or between optical and UV frequencies (e.g. Ghisellini et al. 1986). These can possibly be due to the lack of simultaneity among observations in different bands, or to the reddening which introduces a steepening of the continuum at optical-UV frequencies, or to the contribution of starlight from the host galaxy, which produces a steepening of the energy distribution in the optical and a flattening in the near-IR. If instead the observed spectral breaks are intrinsic to the emission they may be significant to constrain the radiation process.

We report here on quasi simultaneous ( $\Delta \mathrm{t} \lesssim 1$ day) UV, optical and near-IR observations of $11 \mathrm{BL}$ Lac objects obtained in the course of our 10 years systematic multifrequency study of BL Lacs (see e.g. Falomo et al. 1993 and references therein). In two cases (see Table 1) simultaneous IR-optical and optical-UV data taken at different epochs have been combined. UV observations were obtained using both cameras (SWP and LWP) onboard of the International Ultraviolet Explorer (IUE) while optical spectrophotometry and J,H,K, L photometry of the sources were obtained at the European Southern Observatory (ESO). Table 1 gives program objects, along with observation date, redshift, galactic extinction, radio or

T. J.-L. Courvoisier and A. Blecha: Multi-Wavelength Continuum Emission of AGN, 17-20.

C 1994 IAU. Printed in the Netherlands. 
X-ray selection, UV-to-IR spectral slope, percentage contribution and absolute magnitude of the host galaxy.

Table 1: Blazars Observed in UV-Opt-IR

\begin{tabular}{clcccccc}
\hline Objects & Date & $z$ & $A_{V}$ & $\mathrm{R} / \mathrm{X}$ & $\alpha$ & $\% \mathrm{Gal}$ & $\mathrm{M}_{V}^{\dagger}$ \\
\hline $0048-097$ & 87 Jan 7,8 & $\ldots$ & 0.22 & $\mathrm{R}$ & $0.93 \pm 0.02$ & $\ldots$ & $\cdots$ \\
$0118-272$ & 89 Aug 10 & 0.559 & 0.09 & $\mathrm{R}$ & $1.20 \pm 0.01$ & $\ldots$ & $\ldots$ \\
$0301-243$ & 89 Aug 9 & $(0.2)$ & 0.11 & $\mathrm{R}$ & $0.79 \pm 0.02$ & 11 & -22.2 \\
$0323+022$ & 89 Aug 10 & 0.147 & 0.50 & $\mathrm{X}$ & $0.23 \pm 0.04$ & 30 & -22.2 \\
$0414+009$ & 89 Feb 15 & 0.287 & 0.51 & $\mathrm{X}$ & $0.54 \pm 0.05$ & 3 & -21.3 \\
$0422+004$ & 88 Jan 9,10 & $(0.1)$ & 0.42 & $\mathrm{R}$ & $1.20 \pm 0.05$ & 10 & -21.5 \\
$0521-365$ & 87 Jan 8 & 0.055 & 0.21 & $\mathrm{R}$ & $1.43 \pm 0.03$ & 51 & -21.7 \\
$1538+149$ & 88 Aug 2 & 0.605 & 0.20 & $\mathrm{R}$ & $1.34 \pm 0.02$ & $\ldots$ & $\ldots$ \\
$1553+113$ & 88 Aug 2,5 & $\ldots$ & 0.22 & $\mathrm{X}$ & $\dagger \dagger$ & $\ldots$ & $\ldots$ \\
$2005-489$ & 86 Sep/89 Aug & 0.071 & 0.33 & $\mathrm{X}$ & $0.57 \pm 0.02$ & 12 & -22.2 \\
$2155-304$ & 86 Sep/89 Aug & 0.116 & 0.10 & $\mathrm{X}$ & $0.47 \pm 0.02$ & 3 & -22.2 \\
\hline
\end{tabular}

$\dagger$ Values are not K-corrected and computed assuming $\mathrm{H}_{0}=50 ; \mathrm{q}_{0}=0$.

†† IR-Optical region: $\alpha=0.78 \pm 0.02$; UV region: $\alpha=1.56 \pm 0.04$.

\section{Results}

A composite (SFD) was constructed for each object from quasi simultaneous observations. Data were corrected for interstellar reddening using $A_{V}$ of Table 1. In 7 cases we attempted a decomposition of the SFD in terms of a power law plus a standard giant elliptical galaxy. For 2 objects of unknown redshift a rough estimate of $z$ has been assumed based on fit optimization of the galaxy contribution.

In Fig. 1 we report as example the cases of 0048-097, 0323+022, 0422+004, $1553+113$. We found that in all but one case the observed SFD is well accounted for either by a single power law or by a power law plus an elliptical galaxy. The exception is PG 1553+11 whose emission shows a spectral break at $\nu \sim 10^{15} \mathrm{~Hz}$.

The average spectral index of the non thermal component is $\langle\alpha\rangle=0.87 \pm$ 0.41 with a marked tendency for X-ray selected objects to be flatter than radio selected ones (respectively $\langle\alpha\rangle=0.45 \pm 0.15$ and $\langle\alpha\rangle=1.15 \pm 0.24$ ).

The constancy of the UV-to-near-IR spectral shape has been tested (Table 2) comparing the spectral indices in the optical-near-IR region for 33 blazars (Falomo et al. 1993) with the UV spectral indices of 24 sources (Pian and Treves 1993). This constancy is observed also if only common objects (14) are considered and is still maintained subdividing them in $\mathrm{X}$-ray and radio selected subsamples.

The main result that no significant change is present in the shape of BL Lac spectra between their IR-optical part and the UV one is at variance with the 
conclusions reached by other authors who report spectral breaks in the near-IR optical or UV region (e.g. Cruz-Gonzalez and Huchra 1984; Ghisellini et al. 1986; Smith et al. 1987; Ballard et al. 1990). We suggest that this is related to our use of simultaneous data, appropriate correction for reddening and for the host galaxy contribution. The absence of any relevant spectral break indicates that a non thermal synchrotron process from a unique radiating volume is likely responsible for the observed emission.

Table 2: Average Spectral Indices

\begin{tabular}{lcccccc}
\hline & $\left\langle\alpha_{I R O P}\right\rangle$ & $1-\sigma$ & $\mathrm{N}_{o b j}$ & $\left\langle\alpha_{U V}\right\rangle$ & $1-\sigma$ & $\mathrm{N}_{o b j}$ \\
\hline \multicolumn{7}{c}{ Whole samples } \\
All & 1.08 & 0.34 & 33 & 0.97 & 0.41 & 24 \\
X-ray selected & 0.68 & 0.28 & 8 & 0.66 & 0.30 & 10 \\
Radio selected & 1.20 & 0.30 & 25 & 1.20 & 0.33 & 14 \\
\hline
\end{tabular}

Common objects

\begin{tabular}{lcccccc} 
All & 0.92 & 0.41 & 14 & 0.98 & 0.46 & 14 \\
X-ray selected & 0.52 & 0.15 & 6 & 0.66 & 0.39 & 6 \\
Radio selected & 1.22 & 0.22 & 8 & 1.22 & 0.36 & 8 \\
\hline
\end{tabular}

\section{References}

Ballard, K.R., Mead, A.R.G., Brand, P.W.J.L. and Hough, J.H.: 1990, MNRAS, 243, 640 Cruz-Gonzalez, I. and Huchra, J.P.: 1984, AJ, 89, 441

Falomo, R., Bersanelli, M., Bouchet, P. and Tanzi, E. G.: 1993, AJ, 106, 11

Ghisellini, G., Maraschi, L., Tanzi, E.G. and Treves, A.: 1986, ApJ, 310, 317

Impey, C.D. and Neugebauer, G.: 1988, AJ, 95, 307

Landau, R. et al.: 1986, ApJ, 308, 78

Pian, E. and Treves, A.: 1993, to appear in ApJ

Smith, P. S., Balonek, T. J., Elston, R. and Heckert, P. A.: 1987, ApJS, 64, 459 

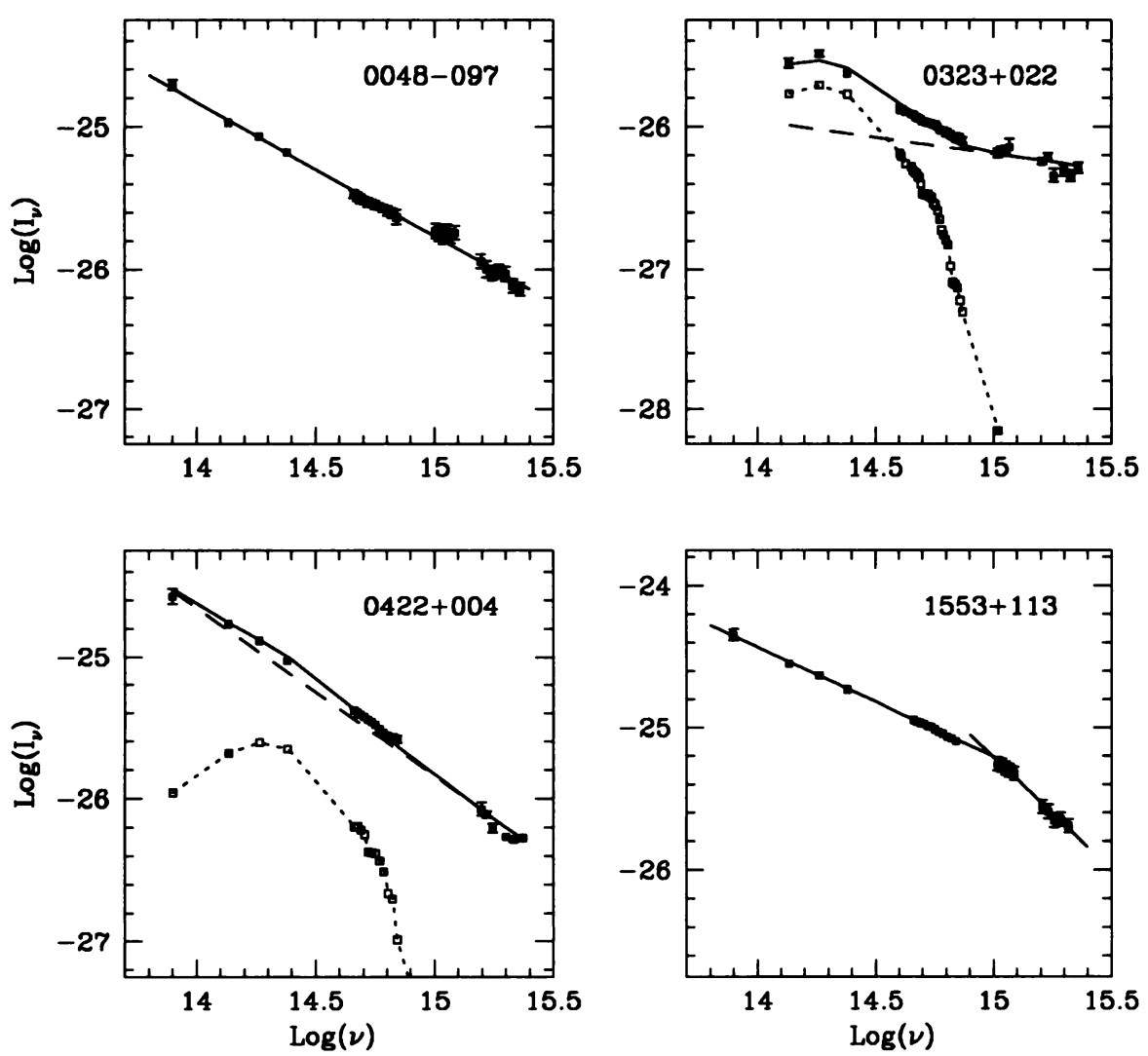

Fig. 1. SFDs of BL Lac objects observed simultaneously at UV, optical and near-IR frequencies. Data (filled squares) are corrected for interstellar extinction. The solid line is the best fit model which is either a single power law or the combination of a host galaxy (dotted line) and a power law component (dashed line). Open squares represent the spectrum of the standard elliptical. 